Document downloaded from:

http://hdl.handle.net/10251/67660

This paper must be cited as:

Seguí Gil, L.; Fito Suñer, PJ.; Fito Maupoey, P. (2013). A study on the rehydration ability of isolated apple cells after osmotic dehydration treatments. Journal of Food Engineering. 115(2):145-153. doi:10.1016/j.jfoodeng.2012.08.038,.

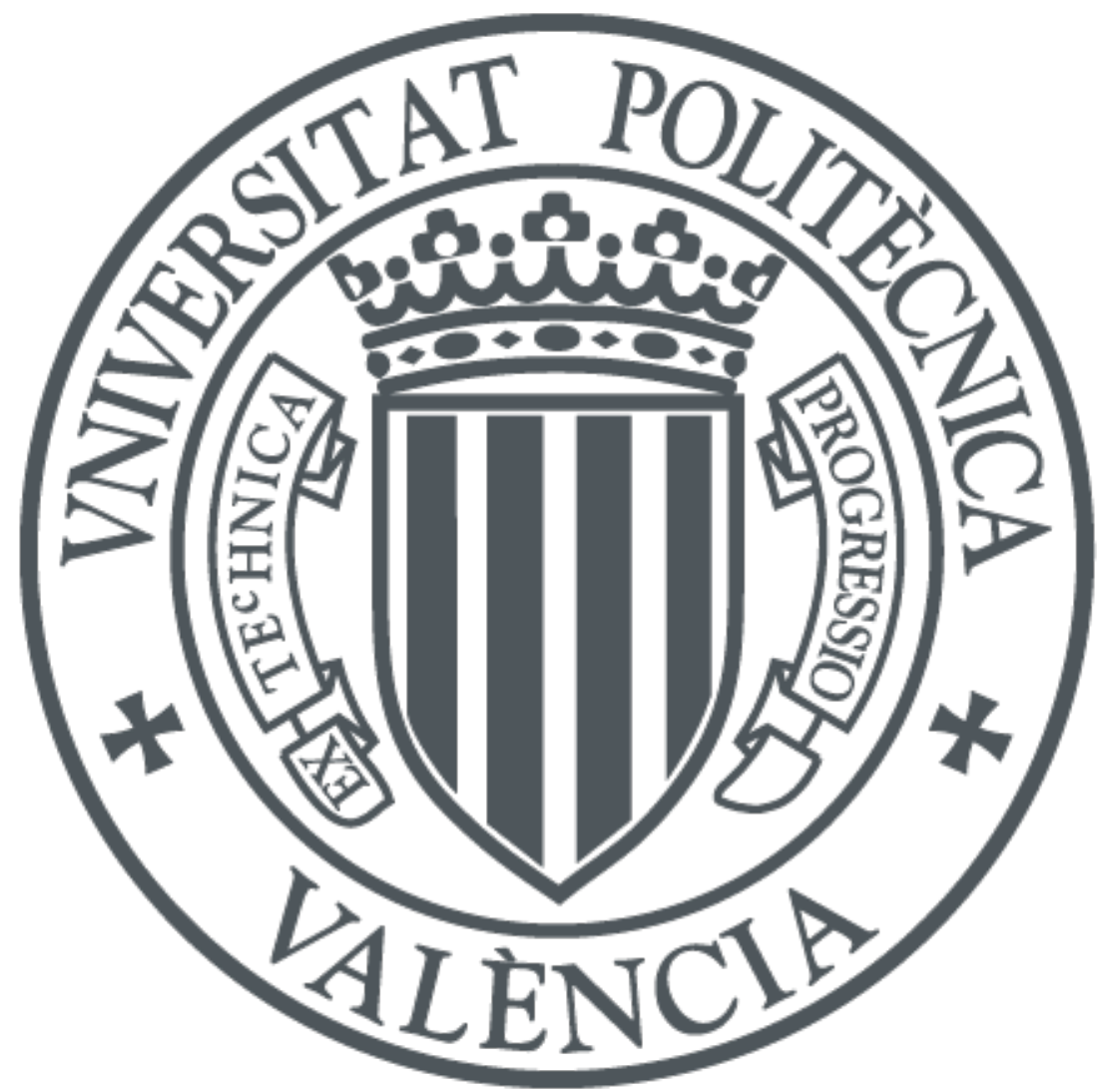

The final publication is available at

https://dx.doi.org/10.1016/j.jfoodeng.2012.08.038

Copyright Elsevier

Additional Information 


\section{ABSTRACT} dehydration.

\section{KEYWORDS}

\title{
A study on the rehydration ability of isolated apple cells after osmotic
} dehydration treatments

\author{
L. Seguí*, P.J Fito, P.Fito
}

Instituto Universitario de Ingeniería de Alimentos para el Desarrollo. Universitat Politècnica de València. Camino de Vera, s/n, Valencia 46022, Spain. Tel.: (0034) 96 3877056; fax: (0034) 96 3877956. E-mail address: lusegil@upvnet.upv.es

A study on rehydration of isolated apple cells is presented. Isolated cells previously dehydrated in $35 \%$ and $25 \%$ sucrose solutions were rehydrated in $5 \%$ sucrose under the microscope with the aim of analysing the phenomena that take place during rehydration. Cells response to rehydration was found to be more heterogeneous than their response to hypertonic treatments. Cells showed different degrees of delay in their response, which was related to differences in the formation and preservation of membrane-to-wall connections. Results confirmed that rehydration success is based on the preservation of the structures along both, dehydration and rehydration treatments. During swelling, Hechtian strands are reincorporated to the protoplast as far as they are formed and preserved during dehydration and rehydration; their absence or shortage leading to a loss of rehydration capacity or even membrane lysis. Different stages have been identified during rehydration, mass transfer being coupled with deformation-relaxation phenomena once the protoplast reaches the cell wall. Phenomenological coefficients for water transfer indicated that rehydration kinetics is faster than water transfer during 
26 Rehydration, osmotic dehydration, isolated cells, mass transfer and deformation-

27 relaxation phenomena.

28

\section{NOMENCLATURE}

30 A projected area, $\left(\mathrm{m}^{2}\right)$.

$31 \mathrm{a}_{\mathrm{j}}$ activity of component $\mathrm{j},(-)$.

$32 \mathrm{~J}_{\mathrm{j}} \quad$ molar flux of component $\mathrm{j},\left(\mathrm{mol} \cdot \mathrm{m}^{-2} \mathrm{~s}^{-1}\right)$.

$33 \mathrm{~K}$ Norrish constant, (一).

$34 \mathrm{~L}$ major axis of equivalent ellipse, (m).

$35 \mathrm{~L}_{\mathrm{j}}$ phenomenological coefficient of component $\mathrm{j},\left(\mathrm{mol}^{2} \cdot \mathrm{J}^{-1} \cdot \mathrm{m}^{-2} \cdot \mathrm{s}^{-1}\right)$.

$36 \mathrm{M}_{\mathrm{rj}} \quad$ molecular weight of component $\mathrm{j},\left(\mathrm{kg} \cdot \mathrm{mol}^{-1}\right)$.

$37 \mathrm{P}$ pressure, $(\mathrm{Pa})$.

$38 \mathrm{R}$ universal gas constant, $\left(\mathrm{J} \cdot \mathrm{mol}^{-1} \cdot \mathrm{K}^{-1}\right)$.

$39 \mathrm{~S}$ surface area, $\left(\mathrm{m}^{2}\right)$.

$40 \mathrm{~T}$ temperature, $(\mathrm{K})$.

$41 \mathrm{t}$ time, (s).

$42 \mathrm{~V} \quad$ volume, $\left(\mathrm{m}^{3}\right)$.

$43 \quad \overline{\mathbf{V}_{\mathbf{j}}} \quad$ partial molar volume of species $\mathrm{j}\left(\mathrm{m}^{3} \cdot \mathrm{mol}^{-1}\right)$.

$44 \mathrm{w}_{\mathrm{j}} \quad$ mass fraction of component $\mathrm{j},\left(\mathrm{kg} \cdot \mathrm{kg}^{-1}\right)$.

$45 \mathrm{x}_{\mathrm{j}} \quad$ molar fraction of component $\mathrm{j},\left(\mathrm{mol} \cdot \mathrm{mol}^{-1}\right)$.

46 Greeks

$47 \quad \rho \quad$ density, $\left(\mathrm{kg} \cdot \mathrm{m}^{-3}\right)$.

$48 \quad \mu_{\mathrm{i}} \quad$ chemical potential of component $\mathrm{i},(\mathrm{J} \cdot \mathrm{mol}-1)$.

49 Subscripts, superscripts and abbreviations.

$50 \quad 0 \quad$ refers to initial conditions. 
51 CW refers to cell or delimited by the cell wall.

52 EP external phase.

53 ext extended

54 IP internal phase.

$55 \mathrm{~N}$ number of cells.

56 OD osmotic dehydration.

57 OS osmotic solution.

58 PM refers to protoplast or delimited by the plasma membrane.

59 ss soluble solids.

$60 \mathrm{t}$ refers to processing time.

61 W water.

\section{INTRODUCTION}

64 Food dehydration causes irreversible damage to the food material. Shrinkage, decrease 65 in porosity, loss of cell compartmentation or changes in physical properties such as 66 texture or colour are common alterations in dried foods. Nevertheless, food dehydration

67 continues to be an interesting preserving operation, not only because it leads to shelf life prolongation and volume reduction, but also as a technique for products diversification

69 and new products design. According to some authors, dehydration could be further 70 expanded if improvements in food quality and process applications are achieved (Atarés 71 et al., 2009; Maskan, 2001). Food dehydration is a widely studied operation: air drying, 72 osmotic dehydration (OD), microwave drying or freeze drying, are some examples. 73 Combinations of different techniques or the use of pretreatments such as OD or vacuum impregnation are also common in the literature. 
Rehydration capacity can be considered as a measure of the damage caused to the food material by dehydration and pretreatments. It is generally accepted that rehydration is intimately related to the degree of cellular and structural damage caused to the food (Krokida et al., 1999; Krokida and Marinos-Kouris, 2003; Krokida and Philippopoulos, 2005; Lewicki, 1998; Sacilik and Elicin, 2006). According to this, the study of rehydration will lead to a better understanding of the changes that the product undergoes during dehydration, and so has been used by others (Witrowa-Rajchert and Lewicki, 2006). The fact that some dehydrated products are eventually consumed rehydrated, e.g. in milk, yoghurt or in instant soups and ready to eat meals, is another important reason for the study of food rehydration processes, since this would be relevant in order to develop this kind of products (Krokida and Philippopoulos, 2005; Prothon et al., 2001). A better understanding of rehydration processes seems to be crucial so as to improve the quality of both dehydrated and rehydrated products, as well as for new products design. Nevertheless, compared to dehydration not much is known about the phenomena undergoing during rehydration. This is true not only from a food engineering point of view, but also from a biological one; according to Lang-Pauluzzi (2000), although the phenomenon of plasmolysis has been extensively studied, there has been less interest in deplasmolysis, and it has been widely assumed that deplasmolysis is the reverse process of plasmolysis. With regard to food engineering, most of the studies published on rehydration focus on the quantification of water absorption and leaching of solutes, and in some of them, kinetics of rehydration is analysed (Krokida and Marinos-Kouris, 2003; Krokida and Philippopoulos, 2005). According to Witrowa-Rajchert and Lewicki (2006), three different phenomena occur during rehydration: the imbibing of water by the dried material, the swelling and the leaching of solutes into the rehydrating medium. Changes in the macroscopic properties of the food have also been referred; however, 
little attention has been paid to microstructural changes during rehydration which, in fact, are essential in order to clarify the process (Moreira et al., 2011; Prothon et al., 2001). Specifically in cellular materials, microstructure is very important since tissue compartmentalization plays a key role for water transfer.

In the present work, an effort has been made in order to identify the phenomena that take place during rehydration at the cellular level. This contribution belongs to a systematic approach focused on the study of osmotic dehydration and rehydration of fruits and vegetables, which general aim consists of better understanding the phenomena that take place at the cellular level, which in turn influence the macroscopic properties of the food. Eventually, some of these microstructural observations or features could be incorporated to the predictive models, which must not only be feasible in predicting water loss or gain, but should ideally be able to predict the macroscopic properties of the food after processing. An increasing interest in microstructural approaches that emphasize the role of the structure in food engineering and models development has been noticed during the last years, in line with the development of the food product engineering concept (Aguilera, 2005; 2006; Ferrando and Spiess, 2002; Fito et al., 2007; Mebatsion et al., 2008, Nieto et al., 2004). Looking at the single elements that build the food may help deduce some of the properties and mechanisms involved in the process, that otherwise are partially misunderstood due, in the case of fruits and vegetables, to tissue complexity. In the present work, isolated cells were chosen as simplified systems so as to segregate the effect of the single cell structure from the effects of the rest of the tissue.

Previous work carried out with isolated apple cells (Seguí et al., 2010; Seguí et al., 2012) showed that cells response to OD depends not only on dehydration rate but also on cells morphology, indicating a clear influence of the structure on the response to 
processing. On the other hand, it was also deduced that more than final water content, the rate at which this water content is achieved is crucial if the structure is to be preserved. Both, morphology and dehydration rate are responsible for the preservation and creation of membrane to wall connections such as the Hechtian structures, which allow the protoplast to be connected with the cell wall after dehydration. According to these results, the ability of a cell to rehydrate is going to be highly dependent on the conditions of the dehydration treatment.

In this work, rehydration of isolated apple cells in diluted sucrose solutions after OD treatments is analysed. The aim of the study was to evaluate the effect of the previous dehydration treatments and of rehydration itself on the cell response, focusing on rehydration ability; as well as to study kinetics of rehydration, at the cellular level.

\section{MATERIALS AND METHODS}

\subsection{Rehydration experiments}

Apple cells (Malus domestica cv. Fuji) were enzymatically isolated from apple parenchyma in a digestion medium containing pectinase and then equilibrated in a manitol solution $\left(\mathrm{a}_{\mathrm{w}}=0.986\right)$ as described in Seguí et al. (2010). Isolated apple cells were dehydrated in a $10 \mathrm{~mL}$ assay tubes containing either 25 or $35 \%$ sucrose solution, during at least 30 minutes; the ratio cells:osmotic solution (OS) being 1:25. Suspensions of the dehydrated cells were examined under a light microscope (DMLM Leica Microsystems) with a CCD camera incorporated which allowed acquiring images for further analysis. Description of dehydrated cells was based on examining four set of images, which resulted in 15 to 20 cells per treatment. Rehydration miniaturized experiments were carried out at constant temperature $\left(30{ }^{\circ} \mathrm{C}\right)$ inside a heating-cooling stage (LTS350, Linkam Scientific Instruments Ltd.) incorporated to the microscope and 
basically consisted of soaking the dehydrated cells in a diluted sucrose solution $(5 \%$ sucrose), acquiring images at increasing time intervals (from $30 \mathrm{~s}$ to $30 \mathrm{~min}$ ) and subsequent treatment and measurement of the images (Adobe Photoshop,v. 7.0; ImageJ, $1.36 \mathrm{~b}$ free version). Measurements consisted of obtaining the projected cross area (A) and major axis (L) of each cell, differentiating between plasma membrane (protoplast) and cell wall delimited areas (PM and $\mathrm{CW}$, respectively); volumes were calculated considering cells as spheroids obtained by rotating the ellipses about their major axis. Projected areas at time zero $\left(\mathrm{A}^{\mathrm{PM}_{0}}, \mathrm{~A}^{\mathrm{CW}}{ }_{0}\right)$ were extrapolated from the $\mathrm{A} v s$. time curves. The response of cells to rehydration was assessed by examining 24 to 27 cells per treatment, whereas measurements along rehydration are the result of 7 repetitions (1 cell/experiment).

\subsection{Kinetics of rehydration. Mass water fluxes.}

Water fluxes across the plasma membrane $\left(\mathrm{J}_{\mathrm{w}}{ }^{\mathrm{PM}}\right)$ were obtained by means of equation 1 , where $\mathrm{V}^{\mathrm{PM}}$ is the cell protoplast volume, $\rho^{\mathrm{IP}}{ }_{\text {ss }}$ the density of the solution inside the protoplast as a function of soluble solids content, $\mathrm{w}^{\mathrm{IP}}{ }_{\mathrm{w}}$ the water mass fraction inside the protoplast, $\overline{\mathrm{S}^{\mathrm{PM}}}$ the mean protoplast surface area, $\Delta \mathrm{t}$ the time interval between two consecutive images, and $\mathrm{Mr}_{\mathrm{w}}$ the water molecular weight. As in previous studies (Seguí et al., 2006; 2012), the solution inside the protoplast was identified as the internal phase (IP), and the solution outside the protoplast as the external one (EP).

$$
J_{w}^{P M}=\frac{-\Delta\left(V^{P M} \cdot \rho_{s s}^{I P} \cdot w_{w}^{I P}\right)}{\overline{S^{P M}} \cdot \Delta t \cdot M r_{w}}
$$

The water mass fraction inside the cell protoplast at initial time $\left(\mathrm{w}^{\mathrm{IP}}{ }_{\mathrm{w} 0}\right)$ was determined from the value of the initial molar water fraction inside the protoplast $\left(\mathrm{x}^{\mathrm{IP}}{ }_{\mathrm{w} 0}\right)$ obtained 
by applying the Norrish equation (Norrish, 1966) to the water activity $\left(a_{\mathrm{w}}\right)$ of the sucrose solution in which the cells had been dehydrated. In the Norrish equation (equation 2), $\mathrm{x}_{\mathrm{w}}$ stands for the molar water fraction, and $\mathrm{K}$ is the Norrish constant (6.47 for sucrose).

$$
a_{w}=x_{w} \exp \left(-K\left(1-x_{w}\right)^{2}\right)
$$

Subsequent water mass fractions were obtained applying the mathematical approach previously developed and already applied to protoplasts (Seguí et al., 2006) and cells (Seguí et al, 2012), which considers the plasma membrane impermeable to solutes and assumes a homogeneous water concentration in both the internal and external phases at each measured time. Since the plasma membrane constitutes the interface, the EP comprises all the solution outside the protoplast.

\section{RESULTS AND DISCUSSION}

\subsection{Examination of cells dehydrated in sucrose solutions.}

Suspension of cells dehydrated in 35 and $25 \%$ sucrose solutions are presented in figure 1a and 1b, respectively. Broken cells or cell debris are not observed since live cells tend to float in hypertonic sucrose solutions; therefore, for dehydration experiments, cells were collected from the top of the assay tube. In figure 1a some cell protoplasts appear spherical and plasmolise centered in the cell, whereas others plasmolise more irregularly, leant to the cell wall and presenting a more polygonal shape. According to previous results (Seguí et al., 2010), the later phenomenon could be due to a higher strength of the local membrane-to-wall connections (stronger anchorage points) together with a scarce creation of Hechtian strands or even to a less elasticity of these, which would be also coherent with the fact that completely plasmolysed cells present a perfect 
197 spherical shape. In figure $1 \mathrm{~b}$ the appearance of cells dehydrated in a $25 \%$ sucrose solution is shown. It can be noticed that protoplasts of cells dehydrated in $25 \%$ sucrose are more rounded than the dehydrated in $35 \%$ sucrose, which have been described before. In order to asses this, protoplast roundness $\left(4 \pi \mathrm{A} /\right.$ perimeter $\left.^{2}\right)$ (Mayor et al., 2008) was measured ( $\mathrm{N}=15$ cells per treatment). Results showed that protoplast roundness was significantly higher in cells dehydrated in the less concentrated osmotic solution: $0.86 \pm 0.03(25 \%)$ vs. $0.79 \pm 0.09(35 \%)$ (p-value $<0.05$, Statgraphics Centurion XVI). Moreover, as can be seen in figure $1 \mathrm{~b}$, rounded protoplasts present a particular shape parallel to the cell wall. This similarity between protoplast and cell wall can be related to a higher formation or preservation of the connections between them, mainly to the formation of elastic Hechtian structures, such as Hechtian strands. On the other hand, it was also observed that cells dehydrated in a more concentrated solution showed a higher incidence of endocytotic vesicles and subprotoplasts which, as quoted by many authors (Gordon and Steponkus, 1984; Oparka et al., 1990; Seguí et al., 2010) appear as a result of a stronger osmotic shock. This was corroborated by analysing $\mathrm{N}=20$ cells/treatment, considering that cells presented an incidence when vesicles or subprotoplasts were clearly identified. Results showed that $60 \%$ of the cells dehydrated in the $35 \%$ sucrose solution had at least one vesicle or subprotoplast $v s .35 \%$ in the case of cells dehydrated in $25 \%$ sucrose. Furthermore, while $45 \%$ of the cells dehydrated in the more concentrated solution presented multiple vesicles $(>4)$, this phenomenon was only observed in one of the cells dehydrated in the $25 \%$ sucrose solution. These differences can also be deduced from figure la,b.

Examining cells at a higher magnification made it possible to observe in some cases a net of strands on the surface of the protoplast (figure 2), which would be an indicator of the formation of Hechtian structures (strands and reticulum). These structures could not 
be observed in all the cells and, even though these where more frequent in cells dehydrated in the less concentrated solution, they were present in cells from both concentrations assayed. Remarkably, the presence of these structures was found to be related to the fact that protoplasts presented a more rounded shape, as the ones in figure 1b, since Hechtian structures were not observed in any polygonal cell.

\subsection{Qualitative analysis of the response of isolated apple cells to rehydration}

During rehydration the higher $\mathrm{a}_{\mathrm{w}}$ of the outer medium promotes a water flux that enters the protoplast through the plasma membrane and causes its swelling. When the protoplast reaches the cell wall, it pushes against it, increasing the pressure and causing cell wall deformation (figures 3, 4). The first thing that was noticed when studying the rehydration of isolated cells was that the response of the cells to rehydration was more heterogeneous than their response to hypertonic treatments, according to previous investigations (Seguí et al., 2010; 2012). It was observed that while some cells reacted almost instantaneously, others had a delay in their response to the hypotonic conditions. According to the mechanosensitive mechanism proposed by Wolfe (Wolfe et al., 1981), protoplast expansion occurs mainly thanks to the incorporation of membrane material to the plasma membrane, the elastic response of the plasmalemma being very short. This membrane material comes from different type of reservoirs such as cytoplasmic vesicles, but a time is needed to activate the mechanisms of membrane material incorporation. On the other hand, cells are able to store the membrane material in a different way since they can use the Hechtian structures (reticulum, strands, threads) as reservoirs, which not only would allow this material to be reincorporated to the membrane during rehydration, but would also allow the membrane to return to specific points in the cell wall (Domozych et al., 2003; Lang-Pauluzzi, 2000; Oparka et al., 
1994). If referring to the whole tissue, this will imply the maintenance of the symplast.

248 According to the different mechanisms that a protoplast may use to reduce its surface area during OD, the disparities observed among cells during rehydration could be due to differences in the formation of the Hechtian structures during dehydration, either in the amount of structures formed or in the degree of breakage (preservation) or elasticity of respond faster to the hypotonic treatment.

In figure 3, the response of two different cells dehydrated in a $35 \%$ sucrose solution these structures. Hence, a delayed response of cells to rehydration would suggest a scarcer formation and/or preservation of Hechtian strands during OD than those that during rehydration in 5\% sucrose is shown. There are clear differences between both cells at the beginning of the rehydration treatment: the first one (figure 3a) presents a completely plasmolysed protoplast, spherical and centered in the cell; whereas the second one (figure $3 \mathrm{~b}$ ) is more oval, not centered but leant to the cell wall, and structures similar to strands or threads can be observed at its surface. According to previous results (Seguí et al, 2010), the response of the cell in figure 3a to dehydration occurs as a result of a relatively high concentration of the OS used but also as a result of the poligonality of the cell, which has several angular sites that would have facilitated protoplast detachment. Along rehydration, its protoplast swells and when reaching the wall, it exerts enough pressure to deform it so that protoplast and cell wall swell together. The cell also reduces its poligonality during the treatment and angular sites smoothen. It must be highlighted here, that between 10 and 30 minutes of rehydration the degree of expansion of the cell decreases, evidencing a relaxation of the structure; moreover, cell turgor is apparently lost, since the protoplast is even detached from the cell wall at the end of the treatment. This fact would be an indicator of a loss of membrane-to-wall connections and, therefore an indicator of irreversible deformations 
occurred during dehydration. With regard to figure $3 \mathrm{~b}$, it can be observed that the intercellular space at $30 \mathrm{~s}$ is significantly smaller than in the cell shown in figure $3 \mathrm{a}$ which would suggest that the non-registered response (first 0-30 seconds) is faster than in the previous case. Besides, the recovery of the cell at the end of rehydration is apparently complete, or at least the detachment of the protoplast is not noticed, suggesting that in this case dehydration is more reversible than before. This, together with a faster response to rehydration, upholds the hypothesis that the creation of Hechtian strands during dehydration, its preservation and higher elasticity, facilitates the further rehydration and consequently, the reversibility of the process.

Light microscopy in the visible range is not a specific technique for the identification of Hechtian structures, since these are very fine structures of living cells that are hardly observable under these conditions (Lang-Pauluzzi, 2000); in fact, if Hecthian structures are not observable, it does not mean that they have not been formed at all but it may be due to a difference in the number, thickness or elasticity of the strands. Hechtian strands thickness may significantly differ depending on the species, cell type, degree of plasmolysis and position in a cell; even within a single cell, strands may change over time, break or coalesce (Lang et al., 2004). Despite not being easily noticeable with the technique used, structures that suggest that the plasma membrane is able to return to specific points located in the cell wall were observed in some cases (figure 4). The image presented here corresponds to the moment at which the main protoplast fuses with a sub-protoplast, which is linked to the cell wall by strands that incorporate to the plasma membrane during deplasmolysis. The same phenomenon was observed by LangPauluzzi (2000) using light-field UV microscopy.

In figure 5 a rehydration sequence of a cell previously dehydrated in a $25 \%$ sucrose solution is shown. A fast response of the cell to the hypotonic treatment is evidenced 
297 since in the first image acquired the protoplast had already reached the cell wall. As in

298 figure $3 \mathrm{a}$, some Hechtian structures may be indentified at the beginning of rehydration,

299 which would confirm that the facility to incorporate membrane material through these

300 structures leads to a faster response. With respect to the preservation of cell turgor

301 pressure after rehydration, protoplast separation is not observed in this case.

302 Ferrando and Spiess (2001) found out that protoplasts and subprotoplasts that appeared 303 during OD acquired a rounded shape (spherical) during rehydration as a result of a 304 decrease in the connections with the cell wall. Similar results had been observed by 305 Lang-Pauluzzi (2000), who identified that rehydration first resulted in further 306 contraction and complete rounding up of the protoplast, and in Hechtian strands 307 disintegration into a line of cytoplasmic droplets. In our experiments, no evidences of 308 Hechtian structures were observed in the most spherical protoplast (Fig. 3a), whereas 309 these were clearly observed in the less spherical one (Fig. 3b), indicating a relationship between the breakage of Hechtian strands and protoplast sphericity during rehydration.

311 Ferrando and Spiess (2001) also observed a turgor loss and a loss of cell viability at the 312 end of the rehydration treatment, based on a weakening of the fluorescence signaling of 313 the protoplast observed by confocal imaging. Besides, they confirmed that cell 314 protoplasts did not completely recover their original volume during rehydration, 315 suggesting a reduction of the available membrane surface during the dehydration 316 process. Likewise, a turgor loss after rehydration has also been evidenced in the present 317 work (Fig. 3a), which would suggest a loss of cell viability or of membrane 318 functionality.

\subsubsection{Classification of the response of isolated cells to rehydration.}


Figure 6 summarizes the response of cells to rehydration as a function of the concentration of the OS used in the previous dehydration treatment. Response is classified as: membrane lysis, loss of functionality or complete rehydration. A different response to rehydration means that different phenomena occur and thus different mechanisms are driving the process. This should be considered for modeling purposes. Membrane lysis increased from 10 to $30 \%$ when increasing the concentration of the OS used in the previous OD treatment from 25 to $35 \%$ sucrose. According to experimental observations, membrane lysis was related to the formation of exocytotic vesicles or subprotoplasts during OD, which are more frequent when a higher concentration of the OS is used, as corroborated in the present and other studies (Gordon and Steponkus, 1984; Oparka et al., 1990; Seguí et al., 2010). The moment at which protoplast and subprotoplast fuses was found to be critical, it many times leading to plasmalemma breakage. An excessive increase in membrane tension is also a reason for membrane lysis, which may occur to cells that do not have a sufficient amount of Hechtian structures to recover the membrane material they have lost during dehydration. These cells typically presented long delayed responses, some of them even being completely unable to incorporate water to the protoplast before bursting.

Loss of functionality stands either for cells that presented a loss of rehydration ability during rehydration (usually 2 to 4 minutes) and for cells that showed a loss of turgor pressure at the end of the treatment. Loss of rehydration ability refers to cells which protoplast stops swelling and, after some seconds, it appears flaccid or cannot swell anymore. This could be due to a low formation of Hechtian structures during OD, which would have forced the protoplast to use other kind of reservoirs such as endocytotic vesicles and, eventually, not being able to continue rehydrating or even lyse. According to Johnson-Fianagan and Singh (1986) and other authors (Ferrando and Spiess, 2001; 
346 Gordon-Kamm \& Steponkus, 1984; Oparka et al., 1990) cytoplasmic vesicles are not usually capable of reincorporating to the membrane during protoplast expansion, this being a reason for membrane lysis in many cases. Although vesicles are supposed to act as protoplast membrane material reservoirs that add to the membrane during swelling (Wolfe, 1986), deformation needs to be done very slowly or, otherwise, membrane tension increases and the plasmalemma eventually breaks or loses its selectivity. Similarly to cells that lysed, these cases usually presented long delayed responses $(>1$ min). Concerning the turgor loss response, it could also be explained by a loss of membrane-to-wall connections. In these cases, the protoplast is unable to return to specific points in the cell wall, evidencing irreversible deformations that cannot be recovered during rehydration. Nevertheless, these cells presented certain ability to rehydrate, the delay in their response being usually within one minute.

According to previous results (Seguí et al., 2010), the formation of Hechtian structures is highly dependent on the rate of change during dehydration, it being not only influenced by the concentration of the osmotic medium but also by the morphology of the cell. More formation and preservation of strands would represent more membrane material available to be incorporated to the protoplast during its swelling; Nevertheless, Ferrando and Spiess (2001) suggested that rehydration rate also influences the ability of cells to reincorporate strands during deplasmolyisis, since these strands may also break during the rehydration process. Likewise, Lang-Pauluzzi (2000) observed that Hecthian strands disintegrate into a line of cytoplasmic droplets at the first stages of rehydration. Thus, it is possible that some cells initially have enough strands to successfully undergo rehydration, but these break as a consequence of rehydration itself, mainly when high rehydration rates are used. The percentage of cells that rehydrate completely was significantly higher in the case of cells previously dehydrated in the $25 \%$ sucrose 
solution ( $70 \%$ vs. $40 \%)$. This is, of course, a consequence of the fact that these cells

372 have been rehydrated to a less extent; nevertheless, it must be reminded that, for a similar degree of water loss, cells dehydrated using higher rates detach more easily from the cell wall and deform it to a less extent, i.e. present a higher breakage of membraneto-wall connections; whereas the ones that dehydrate more slowly preserve their connections and deform together with the cell wall during more time (Seguí et al., 2010).

As an overall conclusion to this analysis it could be stated that, although most dehydrated cells presented a protoplast apparently able to reincorporate water, some of and rehydration processes. accumulated during rehydration and later released when the force that has been deforming the structure stops. In this case, this occurs when the water flux that enters 
deformation imposed to membrane and cell wall; as a result, the energy that has been accumulated is released promoting a water flux out of the protoplast, until equilibrium between forces is reached.

On the contrary, this deformation-relaxation phenomenon is not noticed in the curves that correspond to cells rehydrated from $25 \%$ to $5 \%$ sucrose. According to the previous interpretation, this would mean that in this case the deformation taking place is not elastic and reversible, but viscous and therefore irreversible. Furthermore, comparing the deformation undergone by the cell wall in both cases, it is evidenced that it deforms to a higher degree in cells previously dehydrated in the less concentrated solution, and that this deformation is permanent. As it has been mentioned before, a similar behaviour was identified when studying cells osmotic dehydration (Seguí et al., 2010): permanent deformations of the cell wall are higher when lower osmotic gradients are used. This is related to the fact that a lower dehydration rate allows to better preserve the connections between protoplast and cell wall and, as a consequence, both structures deform together during more time. Along with these results, it could be said that cell wall deformation during rehydration is related to the deformation that the cell wall undergoes during OD, so that during rehydration there is a recovery of the viscous deformations undergone in the previous stage. Time at which the protoplast reaches the cell wall and starts to push against it can also be deduced from figure 7 by examining the cell wall deformation curve. It can be observed that the cell wall starts to deform almost immediately in the case of cells dehydrated in $25 \%$ sucrose, and that it takes at least 2-3 min for cells dehydrated in $35 \%$ sucrose.

The relatively high standard deviation of the values during the first minutes of treatment, mainly in cells previously dehydrated in $35 \%$ sucrose, was a consequence of the heterogeneous response of these cells to the hypotonic treatment. 


\subsection{Kinetics of cell rehydration.}

423

Kinetics of rehydration was studied on cells that had been dehydrated in a $35 \%$ sucrose solution, since these were the only cells that presented a period during which mass transfer was not coupled with the deformation of the cell wall. Likewise, cells that lost their rehydration ability during the treatment were discarded for this analysis.

In figure 8 , mean transmembrane water fluxes of cells rehydrated from $35 \%$ to $5 \%$ sucrose are shown. As compared with previous results (Seguí et al., 2012), rehydration fluxes were greater than the resulting during osmotic dehydration, even if the water activity gradient applied in rehydration was smaller. This suggests that rehydration kinetics is faster than dehydration kinetics and will be discussed next, when analysing the water phenomenological coefficients.

The delay in the response of cells to rehydration, previously identified by examining cells images, is also noticed when quantifying the water flux that enters the protoplast (figure 8). As stated before, cells need to activate the mechanisms to reincorporate the membrane material to the plasma membrane at the beginning of rehydration, thus smaller water fluxes are observed. In cells that underwent a successful complete rehydration, the delay in the response never lasted more than 1 minute and even some of them responded almost immediately; this was most likely due to the fact that these cells had mainly stored membrane material in a form easy to reincorporate, such as Hechtian strands, and not in the form of membrane vesicles. During the first minute, the standard deviation of the points is considerable as a consequence of the heterogeneous behaviour among cells. This heterogeneity was a consequence of the fact that cells needed different times to activate the mechanisms of membrane material reincorporation [15-50 s]; in addition, in some particular cases, cells also showed a sharp increase in the water flux values after the delay, which could be explained by the fact that Hechtian structures 
may allow cells to experiment a fast expansion. After that period, cells showed a more homogeneous behaviour in which, as long as the membrane remains intact, osmosis is the mechanism controlling mass transfer, although mechanisms of membrane reincorporation might modify the process to a certain extent. When the protoplast touches the cell wall (contact time: 2.2-2.8 min), flux values decrease as an evidence of the mechanical resistance of the cell wall to swelling. According to this, contact time will represent a critical point in the rehydration process, since from this moment on the available free energy will not only be used in mass transfer but also in deforming structures and in increasing the pressure that the protoplast exerts against the cell wall.

\subsubsection{Definition of critical points and stages during the rehydration process.}

According to the previous description, cells that rehydrate completely follow three different stages: the first stage would correspond to an induction or delay period and the second and third stages would be separated by the critical point "contact time". Indentifying critical points and stages within a process, allows to deduce the mechanisms involved in each particular stage and, eventually, the equations that should be used to describe them. The stages that a cell undergoes during rehydration can be distinguished in figure 9, where water fluxes are plotted against protoplast deformation $\left(\mathrm{V}^{\mathrm{PM}} / \mathrm{V}^{\mathrm{PM}}\right)$. In this figure, critical points are indicated with a dotted line and correspond to relative protoplast volume at the end of the delay period $\left(\mathrm{V}^{\mathrm{PM}}{ }_{t} / \mathrm{V}^{\mathrm{PM}}{ }_{0}=1.2\right.$, for the longest delay period), and to the relative protoplast volume at contact time $\left(\mathrm{V}^{\mathrm{PM}_{t}} / \mathrm{V}^{\mathrm{PM}_{0}}=1.79 \pm 0.06\right)$

Irreversible thermodynamics have been used to model water transfer in cellular materials (Gekas, 2001; Marcotte et al., 1991; Molz and Ferrier, 1982) and, particularly, they have also been applied to isolated protoplasts and cells (Ferrando and Spiess, 2002; 
472 Seguí et al, 2006; Seguí et al., 2012). In a cellular compartmented system mass transfer 473 is necessarily coupled with mechanical deformations or ruptures of the cellular structure 474 (Fito et al., 2007; Seguí et al., 2012; Oliver et al., 2012) therefore, the extended definition of the chemical potential must be used in this case (Gekas, 2001). According to this, for an isothermal process, the driving force that promotes mass transfer during rehydration is the gradient of the extended water chemical potential (equation 3 ). In this equation, the water chemical potential gradient is given by the compositional and pressure terms. Depending on whether mass transfer is coupled or not with DRP, the pressure term has to be considered or may be neglected.

$$
\Delta \mu_{w}^{e x t}=R T \Delta \ln a_{w}+\overline{V_{w}} \Delta P
$$

During the first stage (delay period), the mechanisms for mass transfer are coupled with mechanisms of plasma membrane reincorporation. In addition, protoplasts may present an elastic response at the beginning of rehydration, although this response is known to be very short (Wolfe, 1981). During this period, reincorporation of membrane material to the protoplast reduces the energy available for mass transfer, and the extended water chemical potential cannot be simplified to the compositional term. According to figure 9 , an increase in $20 \%$ of the protoplast volume is needed to complete this activation process.

During the second stage, as long as the protoplast swells without contacting the cell wall, mass transfer is not coupled with DRP. If assuming that the cell wall is not significantly influencing the transfer of water or solutes, which was corroborated in a previous study (Seguí et al., 2012), equation 3 can be simplified to the compositional term $(\Delta \mathrm{P} \approx 0)$. During the second stage, the driving force of the process is the water activity gradient across the plasma membrane and osmosis the prevailing mechanism for mass transfer. 
497 Regarding the third stage, once the protoplast contacts the cell wall mass transfer is coupled with DRP; in particular, some of the available free energy is used in deforming the cell wall and therefore it is not available for mass transfer. As a consequence, water fluxes reduce, showing a slowing down of the water transfer process. The deformation of the cellular structure has an impact on the pressure term of the extended water chemical potential $(\Delta \mathrm{P}>0)$ which cannot be neglected in this stage.

\subsubsection{Water phenomenological coefficients.}

The phenomenological coefficient that describes water transfer across the plasma membrane $\left(\mathrm{L}_{\mathrm{w}}\right)$ was calculated by fitting experimental results to equation 4 . Equation 4 simplifies the water chemical potential to the compositional term, thus it can only be applied to the periods in which mass transfer is not coupled with DRP. Hence, only points after the time required for the cell to respond to the hypotonic treatment and before protoplast-wall contact $\left(\mathrm{t}^{\mathrm{CONTACT}}\right)$ were fitted to the equation. In figure 10 , an example of the fitting of experimental data to equation 4 is shown. Empty points, not fitted, corresponded to the delay period (right side) and to the moment at which the membrane contacts the cell wall and begins to deform it (left side). The arrow indicates time at which the protoplast contacts the cell wall, as extracted from the images obtained under the microscope.

$$
J_{w}^{P M}=-L_{w} \cdot R T \cdot \ln \left(\frac{a_{w}^{E P}}{a_{w}^{I P}}\right)
$$

Although similar in order, phenomenological coefficients obtained for apple isolated cells during rehydration were slightly higher than the values that these cells presented during osmotic dehydration (Seguí et al., 2012): $1.3 \pm 0.3 \times 10^{-4} \mathrm{~mol}^{2} \cdot \mathrm{J}^{-1} \cdot \mathrm{m}^{-2} \cdot \mathrm{s}^{-1}$ vs. 0.9 $\pm 0.3 \times 10^{-4} \mathrm{~mol}^{2} \cdot \mathrm{J}^{-1} \cdot \mathrm{m}^{-2} \cdot \mathrm{s}^{-1}(\mathrm{p}$-value $<0.1$, Statgraphics Centurion XVI). According to 
these results, kinetics of rehydration is faster than kinetics of dehydration. The differences could be explained taking into account that rehydration phenomenological coefficients have been obtained from cells which swelling has probably been possible thanks to a significant formation and preservation of Hechtian structures, since cells that broke or lost their rehydration ability have been discarded for this analysis. In this way, the plasma membrane material is more easily recovered during protoplast swelling and thus facilitating rehydration. Another possible reason relies on the fact that, according to what has been reported by several authors (Oshima et al., 2001; Ramahaleo et al., 1999; Tazawa et al., 1996), the plasma membrane exhibits a polarity to water transport, this being the reason why the water flux entering the protoplast (endo-osmosis) is usually higher than the water flux going out of it (exo-osmosis). According to these authors, the polarity could be a result of a difference in the selectivity of aquaporins in one or another sense, which would act in favour of the entrance of water in the cell and oppose to cell dehydration.

\section{CONCLUSIONS}

The results obtained in the present work have confirmed that the changes that cells undergo during dehydration determine their ability to rehydrate and, therefore, the characteristics of rehydrated cells. Within a tissue, this is certain to have an impact not only in the rehydration capacity of the product, but also on its macroscopic properties. According to cellular investigations, rehydration success is based on the preservation of the structures along both dehydration and rehydration treatments. Higher osmotic gradients are responsible for membrane lysis during dehydration, but membrane lysis or damage during rehydration is also more frequent in cells previously dehydrated in more concentrated sucrose solutions. It has been deduced that the rate at which changes take 
546 place are crucial in both processes. Success in the reincorporation of strands to the 547 protoplast will depend on the formation and preservation of Hechtian structures during 548 dehydration, but their conservation will also be determined by the rehydration rate. 549 Extrapolating these results to cells in a tissue needs to be done with reservations, since 550 in the whole tissue there are other forces and fluxes acting, but it is expected that 551 reducing osmotic dehydration and rehydration gradients would improve tissue rehydration capacity and, consequently, will have an impact on product quality.

Deformation-relaxation phenomena coupled with mass transfer phenomena have been identified during rehydration. Cells that rehydrate completely undergo three stages

separated by critical points: a delay or induction period in which the mechanisms of membrane material reincorporation are activated, a period during which osmosis is the mechanism that controls mass transfer, and a third stage where mass transfer is coupled with deformations of the cell wall. Considering the extended definition of the chemical potential as the driving force for mass transfer, the pressure term should be considered in the first and third stages and can only be neglected in the second one. Results from this second stage have been used to characterize transmembrane water transfer by calculating the phenomenological coefficients, which have revealed that kinetics of rehydration are faster than dehydration kinetics.

\section{ACKNOWLEDGEMENTS}

The authors would like to acknowledge Ministerio de Educación y Ciencia (Spain) for financial support (Project AGL2009-09905).

\section{REFERENCES}

Aguilera, J.M. (2005). Why food microstructure? Journal of Food Engineering, 67 (12), 3-11. 
Aguilera, J.M. (2006). Seligman lecture 2005 food product engineering: building the right structures. Journal of the Science of Food and Agriculture, 86(8), 1147-1155.

Atarés, L., Chiralt, A. and González-Martínez, C. (2009). Effect of the impregnated solute on air drying and rehydration of apple slices (ct. Granny Smith). Journal of Food Engineering 91, 305-310.

Domozych, D. S., Roberts, R., Danyow, C., Flitter, R. and Smith, B. (2003). Plasmolysis, Hechtian strand formation, and localized membrane-wall adhesions in the desmid, Closterium Acerosum (Chlorophyta). Journal of Phycology, 39, 1194-1206.

Ferrando, M. and Spiess, W.E.L. (2001). Cellular response of plant tissue during the osmotic treatment with sucrose, maltose, and trehalose solutions. Journal of Food Engineering, 49, 115-127.

Ferrando, M. and Spiess, W.E.L. (2002). Transmembrane mass transfer in carrot protoplasts during osmotic treatment. Journal of Food Science, 67 (7), 2673-2680.

Fito, P., LeMaguer, M., Betoret, N. \& Fito, P.J. (2007). Advanced food processing engineering to model real foods and processes: The "SAFES" methodology. Journal of Food Engineering, 83(2), 173-185.

Gekas, V. (2001). Mass transfer modeling. Journal of Food engineering, 49, 97-102.

Gordon-Kamm, W.J. and Steponkus, P.L. (1984). The behaviour of the plasma membrane following osmotic contraction of isolated protoplasts: implications in freezing injury. Protoplasma, 123, 83-94.

Krokida, M.K., Kiranoudis, C.T. and Maroulis, Z.B. (1999). Viscoelastic behaviour of dehydrated products during rehydration. Journal of Food Engineering, 40, 269-277.

Krokida, M. and Marinos-Kouris, D. (2003). Rehydration kinetics of dehydrated products. Journal of Food Engineering, 57, 1-7.

Krokida, M. and Philippopoulos, C. (2005). Rehydration of dehydrated foods. Drying Technology, 23, 799-830.

Lang-Pauluzzi, I. (2000). The behaviour of the plasma membrane during plasmolysis: a study by UV microscopy. Journal of Microscopy, 198, 188-198.

Lang, I., Barton, D.A. and Overall, R.L. (2004) Membrane-wall attachments in plasmolysed plant cells. Protoplasma, 224, 231-24. 
Lewicki, P. (1998). Some Remarks on Rehydration of Dried Foods. Journal of Food Engineering. 36, 81-87.

Marcotte, M., Toupin, C.J. and Le Maguer, M. (1991). Mass transfer in cellular tissues. Part I: The mathematical model. Journal of Food Engineering, 13, 199-220.

Maskan, M. (2001). Drying, shrinkage and rehydration characteristics of kiwifruits during hot air and microwave drying. Journal of Food Engineering, 48 (2), 177-182.

Mayor, L., Pisarra, J. and Sereno A.M. (2008). Microstructural changes during osmotic dehydration of parenchymatic pumpkin tissue. Journal of Food Engineering, 85, 326339.

Mebatsion, H.K., Verboven, P., Ho, A.T. Verlinden, B.E. \& Nicolaï, B.M. (2008). Modelling fruit (micro)structures, why and how. Trends in Food Science and Technology, 19(2), 59-66.

Molz, F.J. and Ferrier, J.M. (1982). Mathematical treatment of water movement in plant cells and tissue: a review. Plant, Cell and Environment, 5, 191-206.

Moreira, R., Chenlo, F., Chaguri, L. and Mayor, L. (2011). of Chestnut Cellular Tissue during Osmotic Dehydration, Air Drying, and Rehydration Processes. Drying Technology, 29: 10-18.

Nieto, A.B., Salvatori, D.M., Castro, M.A. \& Alzamora, S.M. (2004). Structural changes in apple tissue during glucose and sucrose osmotic dehydration: shrinkage, porosity, density and microscopic features. Journal of Food Engineering, 61(2), 269278.

Norrish, R.S. (1966). An equation for the activity coefficients and equilibrium relative humidities of water in confectionery syrups. Journal of Food Technology, 1(25).

Oliver, L., Betoret, N., Fito, P. and Meiders M.B.J. How to deal with visco-elastic properties of cellular tissues during osmotic dehydration. Journal of Food Engineering $110,278-288$.

Oparka, K.J., Prior, D.A.M. and Crawford, J.W. (1994). Behaviour of plasma membrane, cortical ER and plasmodesmata during plasmolysis of onion epidermal cells. Plant, Cell and Enviroment, 17, 163-171. 
Oparka, K.J., Prior, D.A.M. and Harris, N. (1990). Osmotic induction of fluid-phase endocytosis in onion epidermal cells. Planta, 180, 555-561.

Oshima, Y., Iwasaki, I., Suga, S., Murakami, M., Inoue, K. and Masayoshi, M. (2001). Low aquaporin content and low osmótic water permeability of the plasma and vacuolar membranes of a CAM plant Graptopetalum paraguayense: comparison with Radish. Plant Cell Physiology, 42(10), 1119-1129.

Prothon, F., Ahrné, L., Funebo, T., Kidman, S., Langton, M. and Sjöholm, I. (2001). Effects of combined osmotic and microwave dehydration of apple on texture, microstructure and rehydration characteristics. Lebensmittel-Wissenschaft undTechnologie. 34, 95-101.

Ramahaleo, T., Morillon, R., Alexandre, J. and Lassalles, J.P. (1999). Osmotic Water Permeability of Isolated Protoplasts. Modifications during Development. Plant Physiology, 119, 885-896.

Sacilik, K. and Elicin, A.K. (2006). The thin layer drying characteristics of organic apple slices. Journal of Food Engineering, 73 (3), 281-289.

Seguí, L., Fito, P.J., Albors, A.M. and Fito, P. (2007). Mass transfer phenomena during the osmotic dehydration of apple isolated protoplasts (Malus domestica var. Fuji). Journal of Food Engineering, 77 (1), 179-187.

Seguí, L., Fito. P.J. and Fito, P. (2010). Analysis of structure-property relationships in isolated cells during OD treatments. Effect of initial structure on the cell behaviour. Journal of Food Engineering, 99(4), Pages 417-423.

Seguí, L., Fito, P.J. and Fito, P. (2012). Understanding osmotic dehydration of tissue structured foods by means of a cellular approach. Journal of Food Engineering, 110(2), 240-247.

Tazawa, M., Asai, K. and Iwasaki, N. (1996). Characteristics of Hg- and Zn- sensitive

Witrowa-Rajchert, D. and Lewicki, P (2006). Rehydration properties of dried plant tissues. International Journal of Food Science and Technology, 41, 1040-1046.

Wolfe, J. y Steponkus, P.L. (1981). The stress-strain relation of isolated plant protoplasts. Biochimica et Biophysica Acta, 643, 663-668. 